\title{
Effect of changes in sympathovagal balance on the accuracy of heart rate variability obtained from photoplethysmography
}

\author{
XIANG CHEN $^{1-3}$, YUAN-YUAN HUANG ${ }^{1}$, FENG YUN $^{2,3}$, TIAN-JUN CHEN ${ }^{4}$ and JIN LI $^{1}$ \\ ${ }^{1}$ Institute of Biomedical Engineering, School of Life Science and Technology, Key Laboratory of Biomedical \\ Information Engineering of Ministry of Education; ${ }^{2}$ Postdoctoral Mobile Station of Electronic Engineering; \\ ${ }^{3}$ Solid State Lighting Engineering Research Center; ${ }^{4}$ The First Affiliated Hospital, School of Medicine, \\ Xi'an Jiaotong University, Xi'an, Shaanxi 710049, P.R. China
}

Received September 30, 2014; Accepted August 26, 2015

DOI: $10.3892 /$ etm.2015.2784

\begin{abstract}
Heart rate variability (HRV) obtained using photoplethysmography (PPG), which is also known as pulse rate variability (PRV), has already been used in clinical practice. However, it is uncertain whether PRV reflects changes in autonomic nervous function accurately. The aim of the present study was to evaluate quantitatively the effect of alterations in the sympathovagal balance on the agreement between PRV and HRV from electrocardiographs (ECG). Healthy subjects (male, 26; female, 7; age, 22-25 years old) participated in the present study. Paced respiration with 15 breathes/min and breath holding (apnea) were performed to alter the autonomic nervous states of patients. The changes in the low-to-high frequency power ratio $(\mathrm{LF} / \mathrm{HF})$ of $\mathrm{HRV}$ indicated that there was a sympathovagal balance shift toward vagal predominance during paced respiration, but toward sympathetic predominance during apnea. The results demonstrated that, during paced respiration, all indices had an acceptable agreement [Bland-Altman ratio (BAr)<0.2] between PRV and $\mathrm{HRV}$, with the exception of $\mathrm{LF} / \mathrm{HF}$ that had an insufficient agreement $(\mathrm{BAr}=0.25)$. All indices had very strong correlations [Pearson's correlation coefficients $(\mathrm{CC})>0.99$ ] and PRV had a minor but highly significant $(\mathrm{P}<0.001)$ increase for the majority of the variability indices, when compared with HRV. During apnea, the discrepancy of the short-term variability indices between PRV and HRV became sizeable with a BAr $>0.3$ and a minimum $\mathrm{CC}$ of 0.96 . In conclusion, a decrease of $\mathrm{LF} / \mathrm{HF}$ caused a marginal inaccuracy of PRV in the indication of sympathovagal balance, while sympathetic
\end{abstract}

Correspondence to: Professor $\mathrm{Jin} \mathrm{Li}$, Institute of Biomedical Engineering, School of Life Science and Technology, Key Laboratory of Biomedical Information Engineering of Ministry of Education, Xi'an Jiaotong University, 28 Xianning Road, Xi'an, Shaanxi 710049, P.R. China

E-mail: jinlidoc@126.com

Key words: photoplethysmography, pulse rate variability, heart rate variability, sympathetic, vagal balance activation increased differences in short-term variability between PRV and HRV.

\section{Introduction}

Heart rate variability (HRV) analysis is a reliable tool indicating cardiac autonomic modulation, which is useful in the clinical diagnosis of cardiovascular and autonomic diseases (1). Typically, HRV indices calculated based on the RR intervals (RRI) of electrocardiography (ECG), where R is the peak point of the QRS complex, are considered as a clinical standard. In recent years, photoplethysmography (PPG), a simple and ubiquitous technology used to measure the blood volume changes in the microvascular bed, has been widely used in clinical monitoring (2). Since the pulsatile component is synchronous with the heartbeat, PPG has been strongly recommended as an alternative approach to obtain HRV indices.

Due to the variation of pulse transit time (PTT), the pulse intervals of PPG are not exactly the same as the RRIs of ECG. To date, numerous studies have evaluated the agreement between pulse rate variability (PRV) and HRV; however, the results are still disputed $(3,4)$. This uncertainty may be due to deficient methods of analysis or diverse experimental settings. For instance, several studies (5) involved $\leq 4 \mathrm{HRV}$ indices, which is insufficient since $\geq 7 \mathrm{HRV}$ indices ( 2 in the time domain and 5 in the frequency domain) are frequently used in clinical practice (6). In addition, the majority of the existing studies have used Pearson's correlation coefficients (CC) to measure the agreement between PRV and HRV (5); however, $\mathrm{CC}$ reflects the linear correlation instead of the agreement between them, and thus Bland-Altman (BA) analysis may be a more cogent method (7). Furthermore, since HRV is a sensitive indicator reflecting autonomic nervous function, the key point for the accuracy of PRV should be that it accurately indicates the changes in the sympathovagal balance. Experimental conditions must lead to the bidirectional shifts of the sympathovagal balance, which is the shift toward sympathetic predominance and parasympathetic predominance. However, certain studies were only concerned with the accuracy of PRV at resting state (8) or during particular tasks (9). A previous study used activity to alter the autonomic function (10); however, since PPG is susceptible to motion artifacts, the results were not 
fully convincing. It can thus be seen that accurate analysis methods and the appropriate experimental settings are important for evaluating the accuracy of PRV.

In the present study, the use of different respiratory modes to stimulate sympathetic and parasympathetic function was investigated. Initially, the shifts of sympathovagal balance were analyzed. Subsequently, the BA and the CC methods were used to evaluate the agreement between PRV and HRV under different autonomic nervous conditions. The study aimed to achieve a more universal conclusion on the accuracy of HRV from PPG.

\section{Subjects and methods}

Subjects. Experiments were performed on 33 healthy subjects [male, 26; female, 7; median age, 22 years (lower quartile, 22 years; upper quartile, 23 years); age range, 22-25 years]. This study was conducted in accordance with the Declaration of Helsinki and with approval from the Ethics Committee of Xi'an Jiaotong University (Xi'an, China). Written informed consent was obtained from all participants.

Data acquisition. A PPG transducer (TSD 200; Biopac Systems Inc., Goleta, CA, USA) was attached to the left index finger of each individual. The PPG and lead II ECG signals were recorded simultaneously using a multi-channel system (MP 150; Biopac Systems Inc.) at a sampling rate of $1 \mathrm{kHz}$. In addition, a thoracic belt (TSD 201; Biopac Systems Inc.) was used to help the operator judge whether the individuals performed the correct respiratory protocol. The AcqKnowledge software (version 4.2; Biopac Systems Inc.) was used to extract the RRI time series from the ECG signals and to extract the peak-to-peak intervals (PPI) pulse cycle interval time series from the PPG signals. The RRI and PPIs were manually verified beat-by-beat to guarantee detection accuracy. In total, 7,159 beats were obtained during spontaneous respiration, 6,803 beats during paced respiration and 6,755 beats during breath holding.

Experimental protocol. Trials were performed between 2:00 and 5:00 pm. Each subject was asked to refrain from consuming coffee or alcohol from $8 \mathrm{~h}$ prior to the trials. The room was maintained at a temperature of $22 \pm 2^{\circ} \mathrm{C}$. The subjects were instructed to lie in a supine position with their hands comfortably placed at their sides. Subsequent to testing the tolerance to paced respiration and breath holding, 3-min data measurements under each respiratory condition were performed for each subject. Initially, ECG and PPG signals were recorded for $3 \mathrm{~min}$ while the subjects maintained a relaxed state. Next, the subjects followed an audio guide instructing them to breathe with a fixed frequency of 15 breaths $/ \mathrm{min}$, and 3 min signals were recorded. Subsequently, the subjects took a deep breath, held that breath for as long as possible, breathed normally for $30 \mathrm{sec}$, and then began holding their breath again. The same procedure was repeated various times within $3 \mathrm{~min}$, and the signals during normal breathing and breath holding were recorded.

Data processing. For each subject, the RRI and PPI time series were obtained under the three respiratory conditions, and HRV analysis was performed. A total of $11 \mathrm{HRV}$ indices were calculated from the RRI and PPIs, including the following values: Mean value; heart rate (HR), which is the reciprocal of average intervals (mean); SDNN, which is the standard deviation of normal beat intervals; rMSSD, which is the square root of the mean squared differences of successive normal beat intervals; SD1 and SD2, which are the standard deviations of points perpendicular to and along line-of-identity in a Poincaré plot, respectively; LF $\left(\mathrm{msec}^{2}\right)$ and $\mathrm{HF}\left(\mathrm{msec}^{2}\right)$, which are the power values in the low (0.04-0.15 Hz) and high (0.15-0.4 Hz) frequency band ranges, respectively; LF (n.u.) and HF (n.u.), which are the normalized values of LF $\left(\mathrm{msec}^{2}\right)$ and $\mathrm{HF}\left(\mathrm{msec}^{2}\right)$, respectively; and LF/HF, which is the ratio of LF (n.u.) and HF (n.u.) (6).

It has been generally accepted that LF $\left(\mathrm{msec}^{2}\right), \mathrm{SDNN}$ and SD2 represent the long-term variability and are associated with the combination of sympathetic and parasympathetic function. By contrast, HF $\left(\mathrm{msec}^{2}\right)$, rMSSD and SD1 represent the short-term variability and reflect vagus tone, while LF (n.u), HF (n.u) and LF/HF are proportional indices and reflect sympathovagal balance.

Statistical analysis. Data are expressed as the mean \pm standard error. Three statistical analysis methods were used to perform a comprehensive evaluation. A paired-sample t-test was used to assess the difference between the corresponding indices of HRV and PRV. This method was sensitive to the systematic bias, but not to random errors. Pearson's CC was also used to directly measure the correlation between any two indices. In contrast to the t-test, the $\mathrm{CC}$ is sensitive to random errors rather than systematic errors.

The agreements between the HRV and PRV variables were mainly assessed using the BA method (7). BA plots show the differences between PRV and HRV versus the average as the best estimator of the true value. Confidence intervals (CI) for the differences were determined for the mean bias (d) and for the upper (UL) and lower limits (LL) of agreement, using the equations $\mathrm{UL}=\mathrm{d}+1.96 \cdot \mathrm{SD}$ and $\mathrm{LL}=\mathrm{d}-1.96 \cdot \mathrm{SD}$, where $\mathrm{SD}$ is the standard deviation of differences. The $95 \%$ CIs of bias were calculated as $\mathrm{d} \pm \mathrm{t}_{0.05} \cdot \mathrm{SD} / \sqrt{ } \mathrm{n}$. Similarly, the $95 \%$ CIs of the UL and

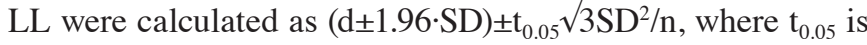
the critical value for a 5\% two-sided test drawn from tables of $\mathrm{t}$ distribution with n-1 degrees of freedom, where $n$ is the sample size. The BA ratio (BAr), which has already been used in a previous study (5), was used to assess the quality of agreement. BA r was defined as BAr=(UL-LL)/2Apm (equation 5), where Apm is the average of the pairwise means. BAr $<0.01$ is considered as an excellent agreement, values between 0.01 and 0.1 are considered as a good agreement, values between 0.1 and 0.2 as a moderate agreement, and values of $>0.2$ are defined as insufficient agreement. The BA plots were produced using MedCalc software, version 12.7.5 (MedCalc Software bvba, Ostend, Belgium).

\section{Results}

Respiration rates. Respiration rates were determined during: i) Spontaneous respiration, which was the normal breathing; ii) paced respiration, with a fixed frequency of 15 breaths/min; and iii) apnea, which was intermittent breath 


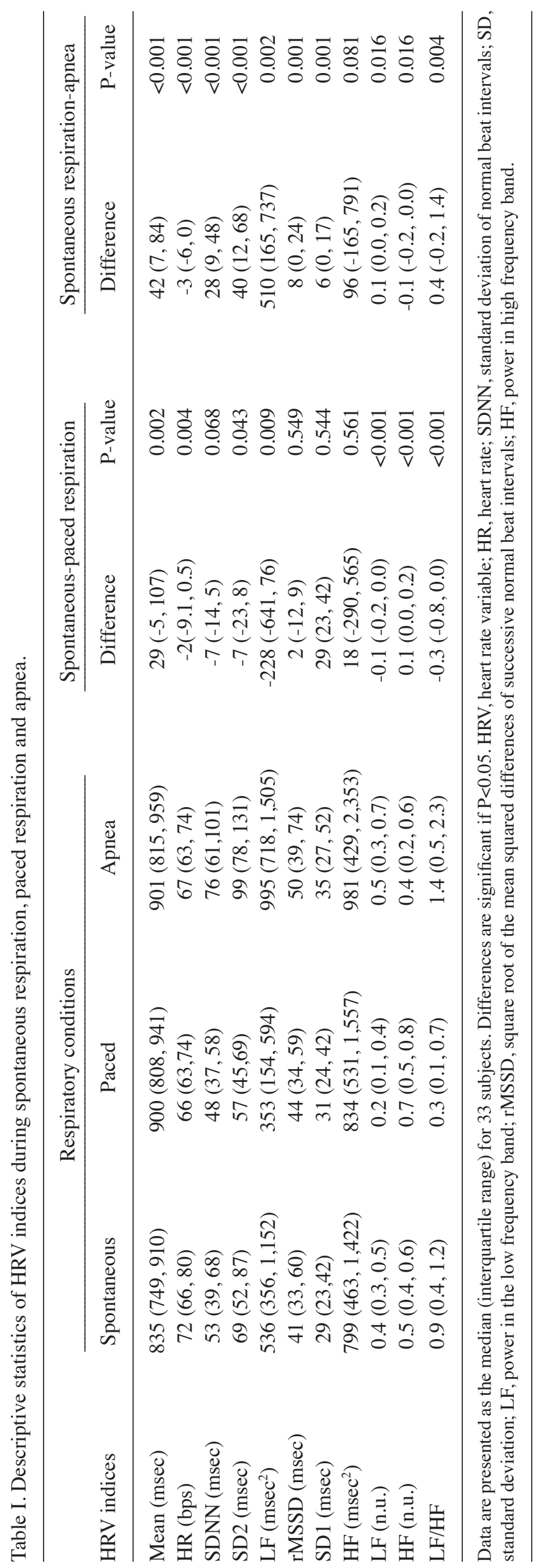

A

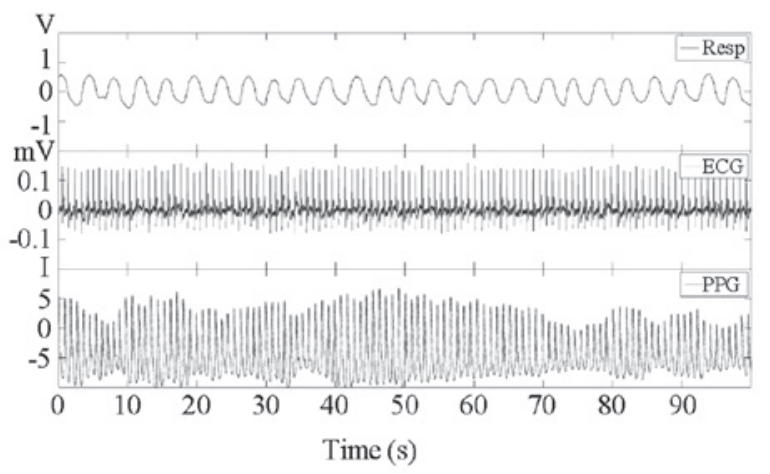

B

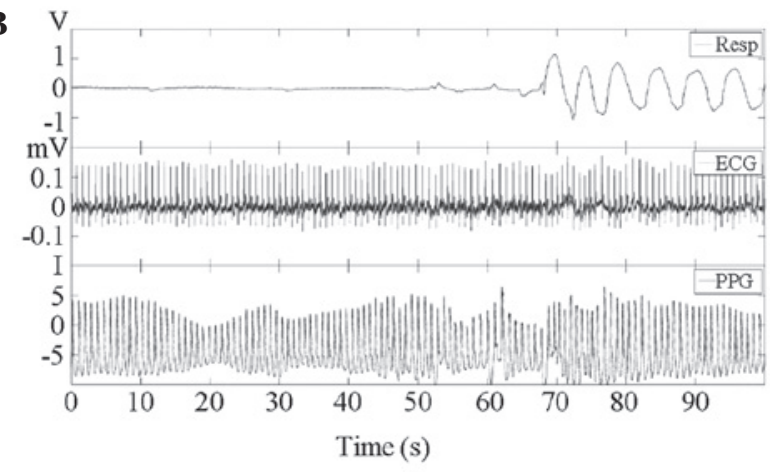

Figure 1. Representative example of the respiratory, ECG and PPG waveforms measured from a subject. (A) Paced respiration at 15 breaths/min; (B) intermittent breath holding. ECG, electrocardiography; PPG, photoplethysmography.

holding. For all subjects, the lowest average respiratory rate was 10 breaths/min, and the highest was 21 breaths/min. The mean respiratory rate of the subjects was $15.2 \pm 3.6$ breaths $/ \mathrm{min}$. Among the 33 subjects, the respiratory rates of 15 subjects was $<15$ breaths/min, and the respiratory rate of the remaining 18 subjects was $>15$ breaths $/ \mathrm{min}$. In addition, the shortest duration of breath holding was $35 \mathrm{sec}$ and the longest duration was $80 \mathrm{sec}$. The mean duration of breath holding for all the subjects was $63.2 \pm 13.2 \mathrm{sec}$.

Representative example of waveform measurements under paced respiration and apnea. A representative example of the waveform measurements from a subject included in the present study is shown in Fig. 1, which includes 90-sec segments of the respiratory, ECG and PPG waveforms. The RRI time series obtained from the ECG signal and PPI time series from PPG are shown in Fig. 2. The amplitude of PPG is the quantified level of intensity.

Descriptive statistics of HRV indices during spontaneous respiration, paced respiration and apnea. The descriptive statistics of HRV indices during spontaneous respiration, paced respiration and apnea are shown in Table I. There were significant differences $(\mathrm{P}<0.05)$ for the indices of the mean, HR, SD2, LF ( $\mathrm{msec}^{2}$ ), LF (n.u.), HF (n.u.) and LF/HF values between paced and spontaneous respiration. In particular, a highly significant decrease $(\mathrm{P}<0.001)$ was observed for the LF/HF ratio from 0.9 (0.4-1.2) during spontaneous respiration to 0.3 (0.1-0.7) during paced respiration, which conventionally implied the shift of sympathovagal balance toward vagal predominance. All other indices, with the exception of 
Table II. Comparison of PRV and HRV indices during paced respiration.

A, CC correlations

\begin{tabular}{|c|c|c|c|c|}
\hline Type of index & Index & PRV & HRV & $\mathrm{CC}$ \\
\hline \multirow[t]{2}{*}{ Heart rate } & Mean (msec) & $890 \pm 26$ & $890 \pm 26$ & $1.00^{\mathrm{a}}$ \\
\hline & HR (bpm) & $69.4 \pm 1.9$ & $69.3 \pm 1.9^{c}$ & $1.00^{\mathrm{a}}$ \\
\hline \multirow[t]{3}{*}{ Long-term variability } & SDNN (msec) & $51.8 \pm 3.6$ & $50.6 \pm 3.5^{\mathrm{a}}$ & $0.99^{\mathrm{a}}$ \\
\hline & SD2 (msec) & $62.9 \pm 4.0$ & $61.4 \pm 3.9^{\mathrm{a}}$ & $0.99^{\mathrm{a}}$ \\
\hline & $\mathrm{LF}\left(\mathrm{msec}^{2}\right)$ & $519 \pm 94$ & $481 \pm 88^{\mathrm{a}}$ & $1.00^{\mathrm{a}}$ \\
\hline \multirow[t]{3}{*}{ Short-term variability } & rMSSD (msec) & $50.8 \pm 5.2$ & $49.2 \pm 5.2^{\mathrm{a}}$ & $0.99^{\mathrm{a}}$ \\
\hline & SD1 (msec) & $36.0 \pm 3.7$ & $34.9 \pm 3.7^{\mathrm{a}}$ & $0.99^{\mathrm{a}}$ \\
\hline & $\mathrm{HF}\left(\mathrm{msec}^{2}\right)$ & $1,489 \pm 366$ & $1,396 \pm 363^{\mathrm{a}}$ & $0.99^{\mathrm{a}}$ \\
\hline \multirow[t]{3}{*}{ Sympathovagal balance } & LF (n.u.) & $0.29 \pm 0.03$ & $0.30 \pm 0.03$ & $0.99^{\mathrm{a}}$ \\
\hline & HF (n.u.) & $0.70 \pm 0.03$ & $0.69 \pm 0.03$ & $0.99^{\mathrm{a}}$ \\
\hline & $\mathrm{LF} / \mathrm{HF}$ & $0.54 \pm 0.09$ & $0.55 \pm 0.09$ & $0.99^{\mathrm{a}}$ \\
\hline
\end{tabular}

$\mathrm{B}, \mathrm{BA}$ ratios

\begin{tabular}{|c|c|c|c|c|}
\hline Index & Bias (CI) & $\begin{array}{l}\text { Lower limits of } \\
\text { agreement }(\mathrm{CI})\end{array}$ & $\begin{array}{l}\text { Upper limits of } \\
\text { agreement }(\mathrm{CI})\end{array}$ & $\mathrm{BA}$ ratio \\
\hline Mean (msec) & $0.01(-0.01$ to 0.04$)$ & $-0.17(-0.23$ to -0.11$)$ & $0.20(0.14$ to 0.26$)$ & $0.00^{\mathrm{d}}$ \\
\hline HR (bpm) & $0.01(0.00$ to 0.02$)$ & $-0.05(-0.07$ to -0.03$)$ & 0.07 (0.05 to 0.09$)$ & $0.00^{\mathrm{d}}$ \\
\hline SDNN (msec) & 1.27 (0.89 to 1.64$)$ & $-0.78(-1.43$ to -0.14$)$ & 3.32 (2.68 to 3.97$)$ & $0.04^{\mathrm{e}}$ \\
\hline SD2 (msec) & 1.45 (1.05 to 1.84$)$ & $-0.74(-1.42$ to -0.05$)$ & 3.64 (2.96 to 4.33$)$ & $0.03^{\mathrm{e}}$ \\
\hline $\mathrm{LF}\left(\mathrm{msec}^{2}\right)$ & $37.2(22.9$ to 51.6$)$ & $-41.9(-66.7$ to -17.2$)$ & $116(91$ to 141$)$ & $0.15^{\mathrm{f}}$ \\
\hline rMSSD (msec) & $1.5(0.9$ to 2.1$)$ & $-1.7(-2.7$ to -0.7$)$ & $4.8(3.8$ to 5.8$)$ & $0.06^{\mathrm{e}}$ \\
\hline SD1 (msec) & $1.1(0.6$ to 1.5$)$ & $-1.2(-1.9$ to -0.5$)$ & $3.4(2.7$ to 4.1$)$ & $0.06^{\mathrm{e}}$ \\
\hline $\mathrm{HF}\left(\mathrm{msec}^{2}\right)$ & $92(57$ to 128$)$ & $-103(-164$ to -41$)$ & 288 (227 to 350$)$ & $0.13^{\mathrm{f}}$ \\
\hline LF (n.u.) & $-0.00(0.01$ to 0.00$)$ & $-0.04(-0.05$ to -0.02$)$ & $0.03(0.02$ to 0.04$)$ & $0.11^{\mathrm{f}}$ \\
\hline HF (n.u.) & $0.00(-0.00$ to 0.01$)$ & $-0.03(-0.04$ to -0.02$)$ & $0.04(0.02$ to 0.05$)$ & $0.05^{\mathrm{e}}$ \\
\hline $\mathrm{LF} / \mathrm{HF}$ & $-0.01(-0.03$ to -0.01$)$ & $-0.14(-0.19$ to -0.10$)$ & 0.12 (0.08 to 0.17$)$ & $-w 0.25^{\mathrm{g}}$ \\
\hline
\end{tabular}

Values are expressed as the mean \pm standard error. Statistical significance: ${ }^{\mathrm{P}}<0.0001$, highly significant; ${ }^{\mathrm{b}} \mathrm{P}<0.01$; ${ }^{\mathrm{c}} \mathrm{P}<0.05$, significant. $\mathrm{B} A$ ratio: ${ }^{\mathrm{d}} \mathrm{BAr}=0.01$, excellent agreement; ${ }^{\mathrm{B} A r}=0.01-0.1$, good agreement; ${ }^{\mathrm{f}} \mathrm{BAr}=0.1-0.2$, moderate agreement; ${ }^{\mathrm{B}} \mathrm{Br}>0.2$, insufficient agreement. $\mathrm{CC}$, correlation coefficient; CI, confidence interval; BA, Bland-Altman HR, heart rate; SD, standard deviation; SDNN, SD of normal beat intervals; $\mathrm{LF}$, power in the low frequency band; rMSSD, square root of the mean squared differences of successive normal beat intervals; HF, power in high frequency band.

$\mathrm{HF}\left(\mathrm{msec}^{2}\right)$, presented statistically significant differences $(\mathrm{P}<0.05)$ between apnea and spontaneous respiration $($ Table I). In particular, the significant increase in the LF/HF ratio implied a sympathovagal balance shift toward sympathetic predominance during apnea. The results indicated the reverse changes in sympathovagal balance during paced respiration and apnea.

$B A$ plots and statistical results of $P R V$ and $H R V$ indices during paced respiration. All long-term and short-term variability indices, including SDNN, SD2, LF ( $\left.\mathrm{msec}^{2}\right)$, rMSSD, $\mathrm{SD} 1, \mathrm{HF}\left(\mathrm{msec}^{2}\right)$ and PRV, presented a highly significant $(\mathrm{P}<0.001)$ increase compared with HRV. In addition, HR showed a statistically significant difference. The indices had strong correlations (CC>0.99) between PRV and HRV. All other indices had an acceptable agreement $(\mathrm{BAr}<0.2)$ between PRV and HRV, with the exception of the ratio of low to high frequency power ( $\mathrm{LF} / \mathrm{HF}, \mathrm{BA} \mathrm{r}=0.25$; Table II). The BA plots for each index during paced respiration are shown in Fig. 3.

$B A$ plots and statistical results of $P R V$ and HRV indices during apnea. Statistically significant differences between PRV and HRV were identified only for LF $\left(\mathrm{msec}^{2}\right)(\mathrm{P}<0.001)$ and SD2 $(\mathrm{P}<0.05)$. The linear correlation was weakened for the short-term variability indices, $r M S S D(C C=0.96)$ and SD1 $(\mathrm{CC}=0.96)$. Furthermore, the agreement between PRV and HRV for all short-term variability indices [rMSSD, SD1 and $\mathrm{HF}\left(\mathrm{msec}^{2}\right)$ ] exceeded the acceptable limit with BAr $>0.3$, but the other indices remained in acceptable agreement $(\mathrm{BAr}<0.2$; Table III). The BA plots for each index under apnea are shown in Fig. 4. 
Table III. Comparison of PRV and HRV indices during Apnea.

A, CC correlations

\begin{tabular}{|c|c|c|c|c|}
\hline Type of index & Index & PRV & HRV & $\mathrm{CC}$ \\
\hline \multirow[t]{2}{*}{ Heart rate } & Mean (msec) & $891 \pm 21$ & $891 \pm 21$ & $1.00^{\mathrm{a}}$ \\
\hline & HR (bpm) & $69.3 \pm 1.6$ & $69.3 \pm 1.7$ & $1.00^{\mathrm{a}}$ \\
\hline \multirow[t]{3}{*}{ Long-term variability } & SDNN (msec) & $85.9 \pm 6.2$ & $85.7 \pm 6.2$ & $0.99^{a}$ \\
\hline & SD2 (msec) & $112 \pm 7$ & $112 \pm 7^{\mathrm{b}}$ & $1.00^{\mathrm{a}}$ \\
\hline & $\mathrm{LF}\left(\mathrm{msec}^{2}\right)$ & $1,464 \pm 249$ & $1,414 \pm 240^{\mathrm{a}}$ & $0.99^{\mathrm{a}}$ \\
\hline \multirow[t]{3}{*}{ Short-term variability } & rMSSD (msec) & $60.9 \pm 6.7$ & $61.7 \pm 6.8$ & $0.96^{\mathrm{a}}$ \\
\hline & SD1 (msec) & $43.2 \pm 4.7$ & $43.7 \pm 4.8$ & $0.96^{\mathrm{a}}$ \\
\hline & $\mathrm{HF}\left(\mathrm{msec}^{2}\right)$ & $1,790 \pm 464$ & $1,762 \pm 446$ & $0.99^{a}$ \\
\hline \multirow[t]{3}{*}{ Sympathovagal balance } & LF (n.u.) & $0.53 \pm 0.03$ & $0.53 \pm 0.03$ & $0.99^{a}$ \\
\hline & HF (n.u.) & $0.46 \pm 0.03$ & $0.46 \pm 0.03$ & $0.99^{\mathrm{a}}$ \\
\hline & $\mathrm{LF} / \mathrm{HF}$ & $1.75 \pm 0.28$ & $1.80 \pm 0.30$ & $0.99^{a}$ \\
\hline
\end{tabular}

$\mathrm{B}, \mathrm{BA}$ ratios

\begin{tabular}{|c|c|c|c|c|}
\hline Index & Bias $(\mathrm{CI})$ & $\begin{array}{l}\text { Lower limits of } \\
\text { agreement }(\mathrm{CI})\end{array}$ & $\begin{array}{l}\text { Upper limits of } \\
\text { agreement }(\mathrm{CI})\end{array}$ & BA ratio \\
\hline Mean (msec) & $0.01(-0.04$ to 0.07$)$ & $-0.32(-0.42$ to -0.21$)$ & $0.35(0.25$ to 0.46$)$ & $0.00^{\mathrm{d}}$ \\
\hline HR (bpm) & $0.00(-0.02$ to 0.02$)$ & $-0.13(-0.18$ to -0.09$)$ & $0.13(0.09$ to 0.18$)$ & $0.00^{\mathrm{d}}$ \\
\hline SDNN (msec) & $0.11(-1.02$ to 1.26$)$ & $-6.22(-8.20$ to -4.24$)$ & $6.45(4.47$ to 8.43$)$ & $0.07^{\mathrm{e}}$ \\
\hline SD2 (msec) & $0.63(0.19$ to 1.07$)$ & $-1.78(-2.53$ to -1.02$)$ & 3.04 (2.29 to 3.80$)$ & $0.02^{\mathrm{e}}$ \\
\hline $\mathrm{LF}\left(\mathrm{msec}^{2}\right)$ & $50(25$ to 76$)$ & $-90(-133$ to -46$)$ & $191(147$ to 235$)$ & $0.09^{\mathrm{e}}$ \\
\hline rMSSD (msec) & $-0.8(-4.3$ to 2.7$)$ & $-20.6(-26.8$ to -14.4$)$ & 19.0 (12.8 to 25.2$)$ & $0.32^{\mathrm{g}}$ \\
\hline SD1 (msec) & $-0.5(-3.1$ to 1.9$)$ & $-14.6(-19.0$ to -10.2$)$ & $13.5(9.1$ to 17.9$)$ & $0.32^{\mathrm{g}}$ \\
\hline $\mathrm{HF}\left(\mathrm{msec}^{2}\right)$ & $27(-81$ to 137$)$ & $-578(-768$ to -389$)$ & 634 (445 to 824$)$ & $0.34^{\mathrm{g}}$ \\
\hline LF (n.u.) & $0.00(-0.00$ to 0.00$)$ & $-0.04(-0.05$ to -0.03$)$ & $0.04(0.03$ to 0.06$)$ & $0.08^{\mathrm{e}}$ \\
\hline HF (n.u.) & $0.00(-0.00$ to 0.00$)$ & $-0.04(-0.06$ to -0.03$)$ & $0.04(0.03$ to 0.05$)$ & $0.09^{\mathrm{e}}$ \\
\hline $\mathrm{LF} / \mathrm{HF}$ & $-0.05(-0.10$ to 0.00$)$ & $-0.35(-0.44$ to -0.25$)$ & $0.24(0.15$ to 0.34$)$ & $0.16^{\mathrm{f}}$ \\
\hline
\end{tabular}

Values are expressed as the mean \pm standard error. Statistical significance: ${ }^{\mathrm{a}} \mathrm{P}<0.0001$, highly significant; ${ }^{\mathrm{b}} \mathrm{P}<0.05$, significant; ${ }^{\mathrm{c}} \mathrm{P}<0.01$, moderately significant. BA ratio: ${ }^{\mathrm{d}} \mathrm{BAr}<0.01$, excellent agreement; ${ }^{\mathrm{e}} \mathrm{BAr}=0.01-0.1$, good agreement; ${ }^{\mathrm{f}} \mathrm{BAr}=0.1-0.2$, moderate; ${ }^{\mathrm{g}} \mathrm{BAr}>0.2$, insufficient agreement. CC, correlation coefficient; CI, confidence interval; BA, Bland-Altman; HR, heart rate; SD, standard deviation; SDNN, SD of normal beat intervals; LF, power in the low frequency band; rMSSD, square root of the mean squared differences of successive normal beat intervals; HF, power in high frequency band.
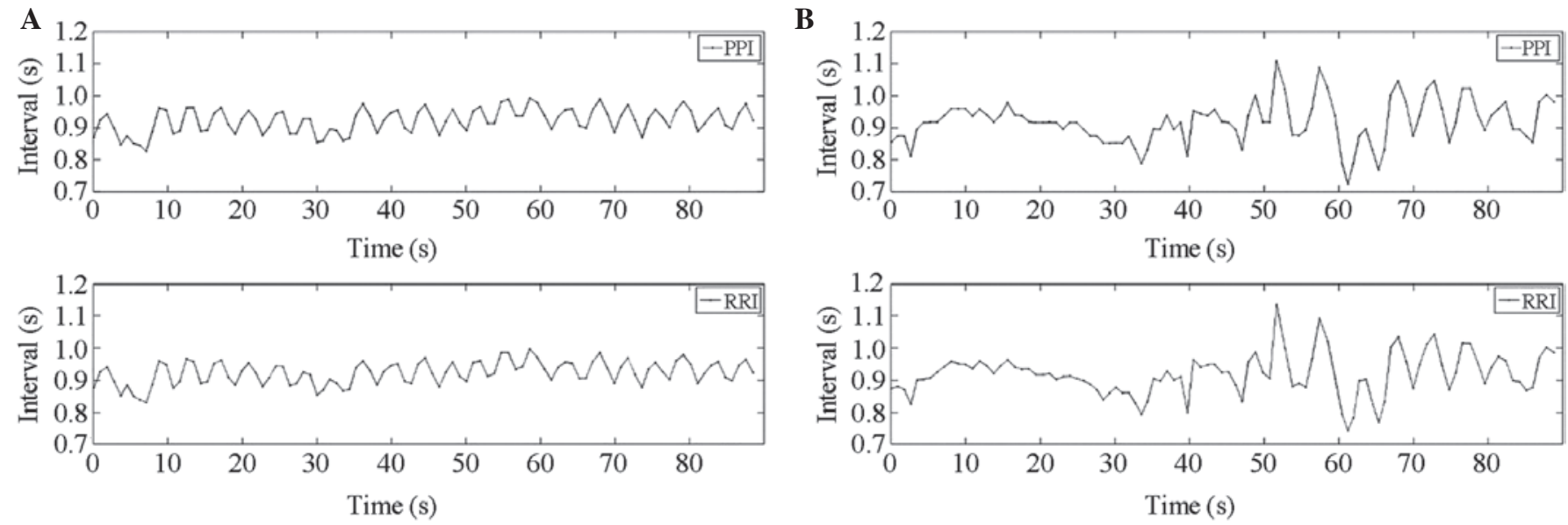

Figure 2. RR interval time series from ECG and PP interval time series from PPG waveforms in Fig. 1. (A) Paced respiration at 15 breaths/min; (B) breath holding. ECG, electrocardiography; PPG, photoplethysmography. 

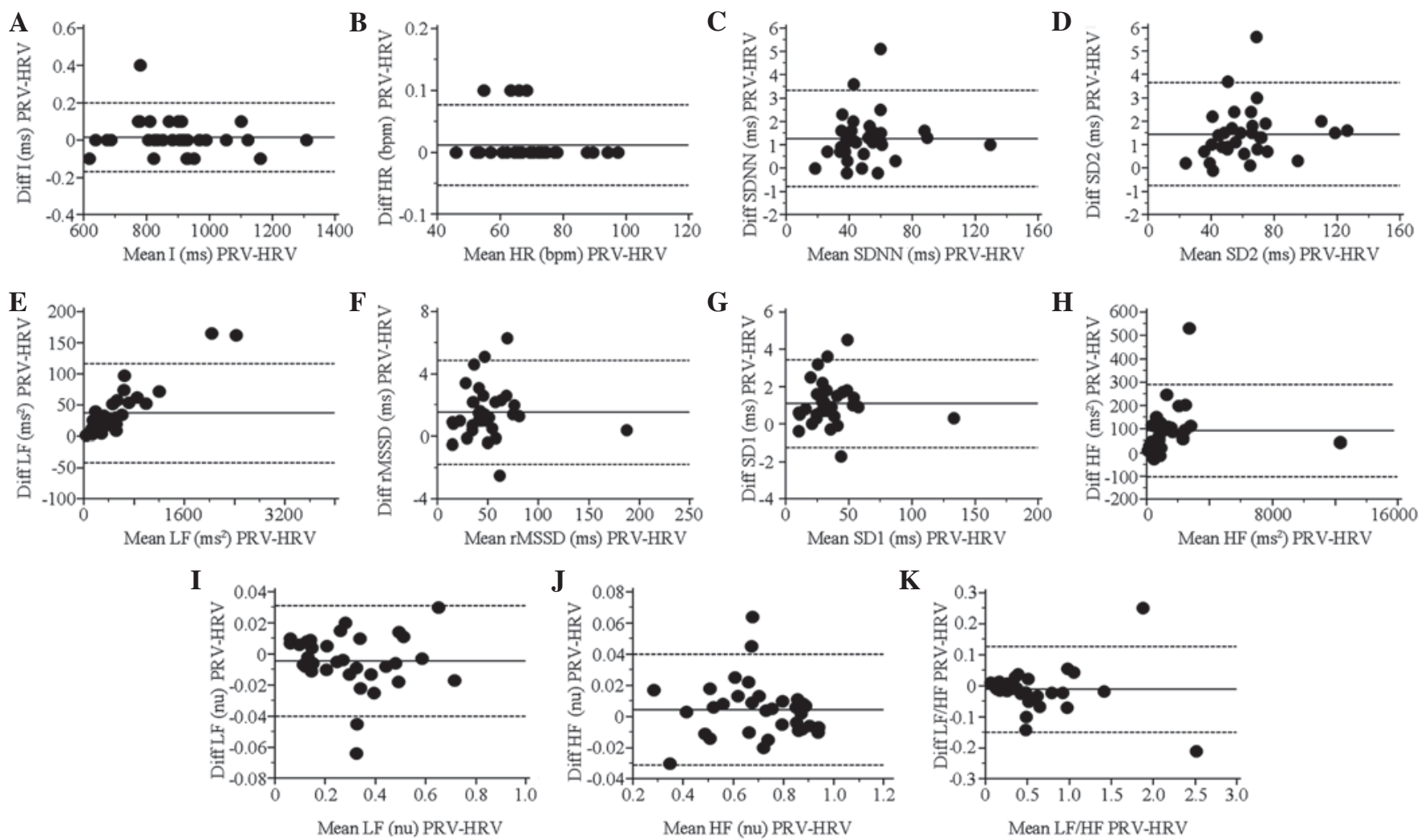

Figure 3. Bland-Altman plots between PRV and HRV for each index during paced respiration. (A) Mean interval; (B) HR; (C) SDNN; (D) SD2; (E) LF; (F) rMSSD; (G) SD1; (H) HF; (I) LF; (J) HF; (K) LF/HF ratio. The solid line shows the mean, while the dotted lines indicate the upper and lower limits. PRV, pulse rate variability; HRV, heart rate variability; SD, standard deviation; SDNN, SD of normal beat intervals; LF, power in the low frequency band; rMSSD, square root of the mean squared differences of successive normal beat intervals; HF, power in high frequency band.
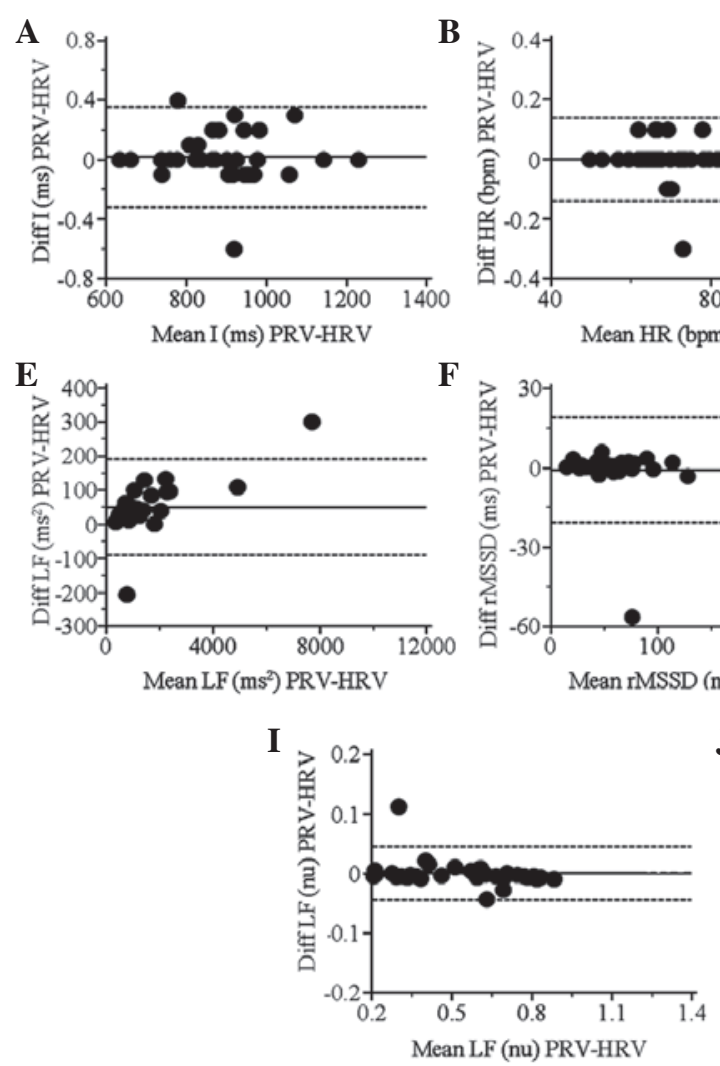
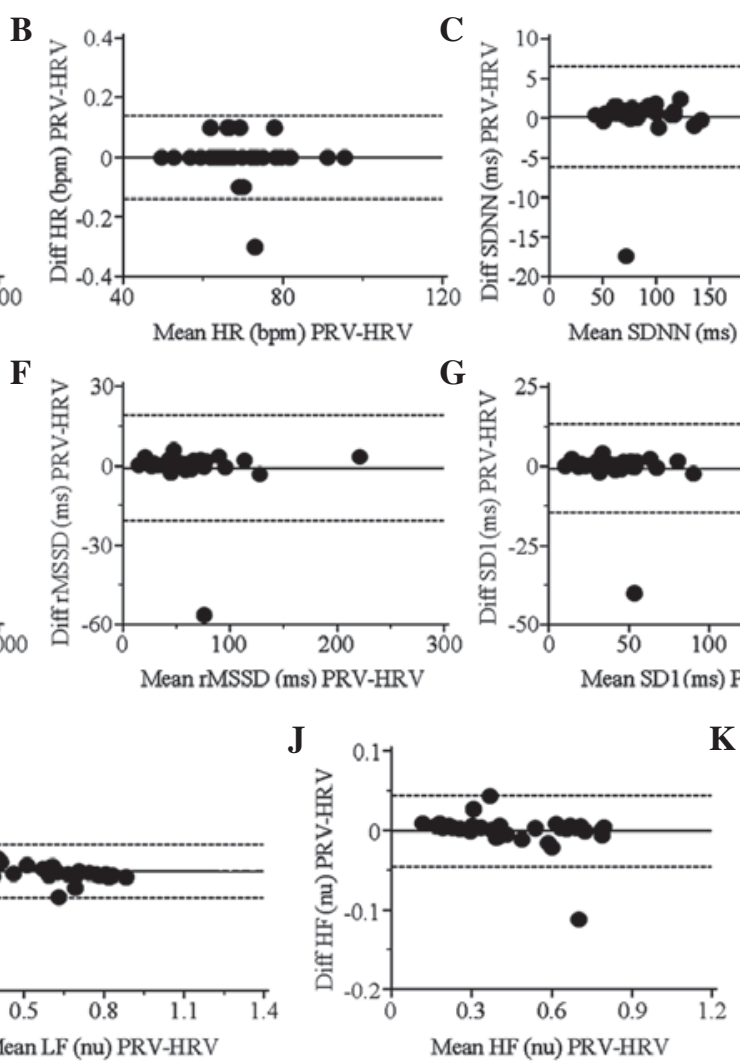
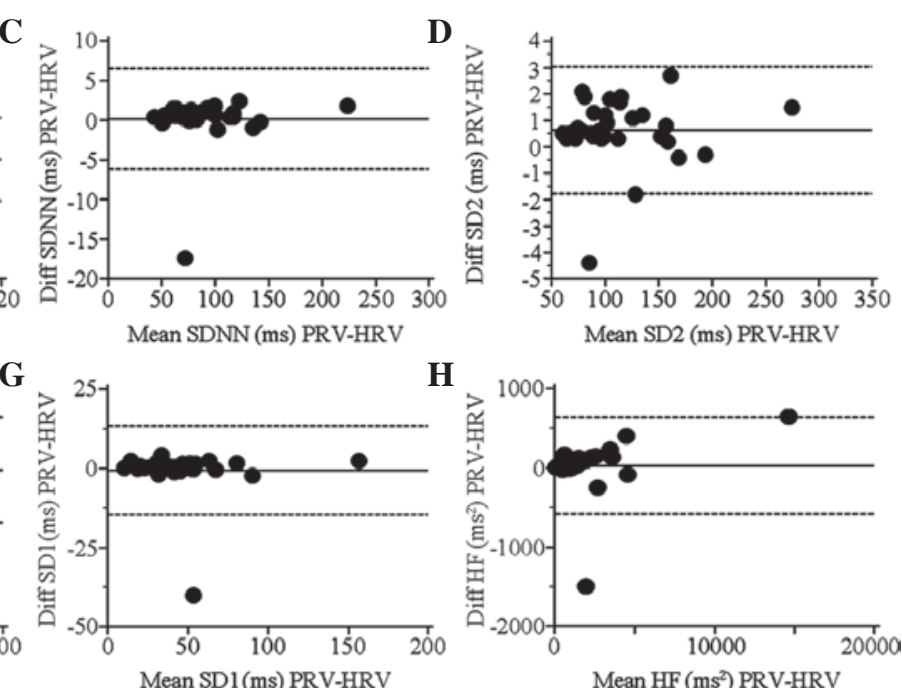

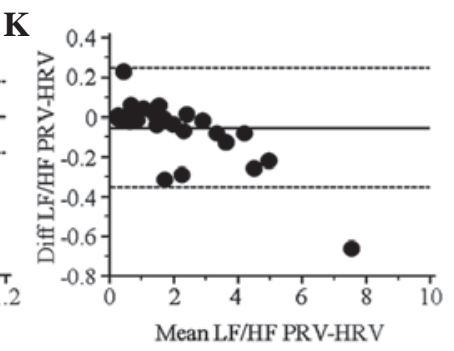

Figure 4. Bland-Altman plots between PRV and HRV for each index during apnea: (A) Mean interval; (B) HR; (C) SDNN; (D) SD2; (E) LF; (F) rMSSD; (G) SD1; (H) HF; (I) LF; (J) HF; and (K) LF/HF ratio. The solid line shows the mean, while the dotted lines indicate the upper and lower limits. PRV, pulse rate variability; HRV, heart rate variability; SD, standard deviation; SDNN, SD of normal beat intervals; LF, power in the low frequency band; rMSSD, square root of the mean squared differences of successive normal beat intervals; HF, power in high frequency band. 


\section{Discussion}

Due to deficient methods of analysis and the diverse experimental settings, the accuracy of HRV obtained from PPG is mostly incommensurable (5). The present study aimed to achieve a more universal conclusion on the accuracy of PRV. Different respiratory modes were used to stimulate the sympathetic and parasympathetic functions. The effect of respiratory-induced changes in sympathovagal balance on the agreement between PRV and HRV was then evaluated. Changes in the LF/HF of HRV indicated that there was a sympathovagal balance shift toward vagal predominance during paced respiration, but toward sympathetic predominance during apnea. In addition, the results demonstrated that during paced respiration, the other indices had an acceptable agreement $(\mathrm{BAr}<0.2)$ between PRV and HRV, with the exception of LF/HF that showed an insufficient agreement (BA $r=0.25)$. The indices had very strong correlations $(C C>0.99)$ and $P R V$ had a highly significant $(\mathrm{P}<0.001)$ increase for the majority of the variability indices, when compared with HRV. During apnea, the agreement of the indices reflecting short-term variability (rMSSD, SD1 and HF) was reduced and was below the acceptable limit, although statistically significant differences only existed in the SD2 and LF values between PRV and HRV.

The present study revealed that paced respiration resulted in the shift of the sympathovagal balance toward vagal predominance, and this result differed from the findings of a previous study indicating that no significant changes were observed in the spectral indices of HRV during paced breathing at $0.25 \mathrm{~Hz}$ (11). The different findings may be due to the different physiological conditions. In the current study, the data during paced respiration were recorded in the first $3 \mathrm{~min}$ of the paced respiration. The subjects were in the adaptation of the new respiratory state, during which the cardiopulmonary and autonomic nervous systems were in the process of establishing a new equilibrium state. By contrast, in the previous study (11), the subjects were trained to adapt the paced respiration prior to the data measurement and, in order to make the subjects comfortable, an adjustment within $\pm 10 \%$ of $0.25 \mathrm{~Hz}$ respiratory frequency was used, while the data were recorded for $8 \mathrm{~min}$. Thus, the autonomic nervous system was possibly much closer to a relatively stable state. However, other contradictory results have also been observed in previous studies, where the paced breathing increased parasympathetic activity during a $\leq 5 \mathrm{~min}$ time period $(12,13)$.

The beat-to-beat variability of PTT is primarily responsible for the possible difference between PRV and HRV. It has been verified that PTT variation had an inversely proportional association with changes in blood pressure, which may result from the autonomic regulation on HR, cardiac contractility, vascular resistance and compliance (14). For spontaneous respiration at resting, during which sympathetic and vagal branches are in a relatively balanced state, PTT variability keeps a relative consistency with HRV; thus, PRV and HRV show good agreement for all HRV indices, as previous studies stated $(15,16)$. However, the paced respiration produced decreases in sympathetic activation that would lead to a reduction in variability of PTT (17). Based on this notion, the random error due to PTT variation decreases, and the difference between PRV and HRV indices tends to be constant. In the present study, the systematic discrepancy caused by different measurements became predominant, but remained within an acceptable limit. In the present study, a significant difference for long-term and short-term indices was observed between PRV and HRV and at the same time, BA $r$ for most indices was $<0.2$ during paced respiration. The only exception was the LF/HF ratio that showed an insufficient agreement (BAr=0.25), which may be due to the notable decrease in the value of LF/HF. Specifically, the average of the pairwise means Apm during paced respiration became lower, so even if there was no increase in UL-LLr, (UL-LL)/2Apm was still high [see equation 5].

By contrast, apnea resulted in sympathetic predominance. Based on a previous study (17), the enhancement of sympathetic activity during breath holding should have led to an increase in the beat-to-beat variability of PTT. Accordingly, the beat-to-beat discrepancy between RRI and PPI increased. Thus, the indices associated with short-term variability, including rMSSD and SD1 and HF, exhibited an insufficient agreement (BAr $>0.2)$. However, the beat-to-beat minor differences had no significant impact on the overall characteristics of PRV, and thus the other indices during apnea had an acceptable agreement between PRV and HRV.

The results from healthy subjects during apnea differed from patients with sleep apnea. A previous study stated that the short-term (2 min) variability indices (rMSSD and HF), the long-term variability index (SDNN) and the index associated with sympathovagal balance (LF/HF) between PRV and HRV showed significant differences during the obstructive sleep apnea events (18). Patients with sleep apnea usually develop circulatory disorders, such as arrhythmia, hypertension and heart failure (19), which may alter the PPG pulse characteristics (2). Thus, the pathological changes may lead to the different results. Nevertheless, the results from healthy subjects provide a reference for using PPG in sleep apnea and other diseases.

In conclusion, the results demonstrated that HR indices (mean and HR values), long-term variability indices (SDNN, SD2 and LF) and two proportional indices reflecting sympathovagal balance of PRV (LF and HF) offer stable reliability regardless of respiratory mode. However, the agreement between PRV and HRV in terms of short-term variability (rMSSD, SD1 and HF) and LF/HF is susceptible to respiratory changes. Additionally, compared with spontaneous respiratory conditions, HRV presented significant and reversible changes under paced respiratory and apnea conditions. Such opposite changes in HRV reflect the inverse autonomic nervous responses to different respiratory ways. Therefore, a more general conclusion may be obtained on the accuracy of PRV for healthy subjects. When long-term variability and sympathovagal balance increase significantly, the agreement for short-term variability indices between PRV and HRV would become insufficient, while the significant decrease of long-term variability and sympathovagal balance may result in a marginal inaccuracy of LF/HF. The results of this study presented the distinctive characteristics of PRV indices in different autonomic nervous states, which should be considered when applying PPG as an alternative approach of ECG to obtain HRV indices for the evaluation of autonomic function. 


\section{Acknowledgements}

This study was supported by grants from the National Natural Science Foundation of China (no. 81101117), the Natural Science Foundation of Shaanxi Province (no. 2012JM4030), the Shaanxi Province Foundation for Returnees (no. SLZ2009008), the Fundamental Research Funds for the Central Universities (nos. XJJ20100170, XJJ2012129 and 2012JDHZ49) and the China Postdoctoral Science Fund (no. 2012M521779).

\section{References}

1. Kemp AH and Quintana DS: The relationship between mental and physical health: Insights from the study of heart rate variability. Int J Psychophysiol 89: 288-296, 2013.

2. Allen J: Photoplethysmography and its application in clinical physiological measurement. Physiol Meas 28: R1-R39, 2007.

3. Wong JS, Lu WA, Wu KT, Liu M, Chen GY and Kuo CD: A comparative study of pulse rate variability and heart rate variability in healthy subjects. J Clin Monit Comput 26: 107-114, 2012

4. Gil E, Orini M, Bailón R, Vergara JM, Mainardi L and Laguna P Photoplethysmography pulse rate variability as a surrogate measurement of heart rate variability during non-stationary conditions. Physiol Meas 31: 1271-1290, 2010.

5. Schäfer A and Vagedes J: How accurate is pulse rate variability as an estimate of heart rate variability? A review on studies comparing photoplethysmographic technology with an electrocardiogram. Int J Cardiol 166: 15-29, 2013.

6. Heart rate variability: Standards of measurement, physiological interpretation and clinical use. Task force of the European Society of Cardiology and the North American Society of Pacing and Electrophysiology. Circulation 93: 1043-1065, 1996.

7. Bland JM and Altman DG: Statistical methods for assessing agreement between two methods of clinical measurement. Lancet 1: 307-310, 1986.
8. Lu G, Yang F, Taylor JA and Stein JF: A comparison of photoplethysmography and ECG recording to analyse heart rate variability in healthy subjects. J Med Eng Technol 33: 634-641, 2009.

9. Giardino ND, Lehrer PM and Edelberg R: Comparison of finger plethysmograph to ECG in the measurement of heart rate variability. Psychophysiology 39: 246-253, 2002.

10. Charlot K, Cornolo J, Brugniaux JV, Richalet JP and Pichon A: Interchangeability between heart rate and photoplethysmography variabilities during sympathetic stimulations. Physiol Meas 30: 1357-1369, 2009.

11. Pinna GD, Maestri R, La Rovere MT, Gobbi E and Fanfulla F: Effect of paced breathing on ventilatory and cardiovascular variability parameters during short-term investigations of autonomic function. Am J Physiol Heart Circ Physiol 290: H424-H433, 2006.

12. Driscoll D and Dicicco G: The effects of metronome breathing on the variability of autonomic activity measurements. J Manipulative Physiol Ther 23: 610-614, 2000.

13. Stark R, Schienle A, Walter B and Vaitl D: Effects of paced respiration on heart period and heart period variability. Psychophysiology 37: 302-309, 2000.

14. Foo JY and Lim CS: Pulse transit time as an indirect marker for variations in cardiovascular related reactivity. Technol Health Care 14: 97-108, 2006.

15. Hayano J, Barros AK, Kamiya A, Ohte N and Yasuma F: Assessment of pulse rate variability by the method of pulse frequency demodulation. Biomed Eng Online 4: 62, 2005.

16. Shi P, Hu S and Zhu Y: A preliminary attempt to understand compatibility of photoplethysmographic pulse rate variability with electrocardiogramic heart rate variability. J Med Biol Eng 28: 173-180, 2008.

17. Malliani A, Pagani M, Lombardi F and Cerutti S: Cardiovascular neural regulation explored in the frequency domain. Circulation 84: 482-492, 1991.

18. Khandoker AH, Karmakar CK and Palaniswami M: Comparison of pulse rate variability with heart rate variability during obstructive sleep apnea. Med Eng Phys 33: 204-209, 2011.

19. Leung RS: Sleep-disordered breathing: Autonomic mechanisms and arrhythmias. Prog Cardiovasc Dis 51: 324-338, 2009. 